\title{
Brain-Derived Neurotrophic Factor (Val66Met) Polymorphism Does Not Influence Recovery from a Post-Traumatic Vegetative State: A Blinded Retrospective Multi-Centric Study
}

\author{
Sergio Bagnato,,2 Luigi Minafra, ${ }^{3,4}$ Valentina Bravatà,, ${ }^{3,4}$ Cristina Boccagni, ${ }^{1,2}$ Antonino Sant'Angelo,, \\ Alberto Castiglione, Maria Andriolo, ${ }^{5}$ Lucia Francesca Lucca, ${ }^{6}$ Antonio De Tanti, ${ }^{7}$ Caterina Pistarini, ${ }^{8}$ \\ Rita Formisano, ${ }^{9}$ Giuliano Dolce, ${ }^{6}$ Cecilia Gelfi, ${ }^{4,10}$ and Giuseppe Galardi ${ }^{1,2}$
}

\begin{abstract}
Brain-derived neurotrophic factor (BDNF) is a neurotrophin that influences neuronal plasticity throughout life. Emergence from a vegetative state (VS) after a traumatic brain injury (TBI) implies that the brain undergoes plastic changes. A common polymorphism in the BDNF gene-BDNF Val66Met (referred to herein as $\mathrm{BDNF}_{\mathrm{Met}}$ )-impairs cognitive function in healthy subjects. The aim of this study was to determine whether the $\mathrm{BDNF}_{\mathrm{Met}}$ polymorphism plays a role in the recovery of consciousness and cognitive functions in patients in a VS after a TBI. Fifty-three patients in a VS 1 month after a TBI were included in the study and genotyped for the BDNF $_{\text {Met }}$ polymorphism. Scores of levels of cognitive functioning (LCF) at 1, 3, 6, and 12 months post-TBI were retrospectively compared in patients without (Val group), and with (Met group), the BDNF Met $_{\text {polymorphism. }}$ The $\mathrm{BDNF}_{\text {Met }}$ polymorphism was detected in 20 out of the 53 patients. The mean LCF scores in the Val and Met groups were $1.6 \pm 0.5$ and $1.4 \pm 0.5$ at 1 month, $2.3 \pm 0.7$ and $2.5 \pm 1.2$ at 3 months, $3.3 \pm 1.7$ and $3.5 \pm 1.7$ at 6 months, and $4 \pm 1.9$ and $3.9 \pm 1.8$ at 12 months, respectively $(p>0.05)$. The percentages of patients in the Val and Met groups who emerged from the VS were $36.4 \%$ and $30 \%$ at 3 months, $66.3 \%$ and $70 \%$ at 6 months, and $70 \%$ and $87.5 \%$ at 12 months $(p>0.05)$, respectively. These findings provide evidence that the BDNF Met $_{\text {polymor- }}$ phism is not involved in cognitive improvement in patients with a VS following TBI. Future studies should focus on the role of other BDNF polymorphisms in the recovery from a VS.
\end{abstract}

Key words: disorders of consciousness; genetic factors; levels of cognitive functioning; traumatic brain injury; vegetative state

\section{Introduction}

A FTER A PERIOD OF COMA due to a severe traumatic brain injury (TBI), patients who do not recover consciousness or die develop a dramatic condition known as vegetative state (VS). The hallmark feature of a VS is a dissociation between the two cardinal elements of consciousness: awareness and wakefulness. Indeed, patients in a VS seem to be awake, but lack any sign of awareness of themselves or of their environment (Monti et al., 2010). The three main factors associated with recovery from a VS include: (1) the type of brain injury, (2) the patient's age, and (3) the time spent in a VS (Monti et al., 2010; Royal College of Physicians, 2003; The MultiSociety Task Force on PVS, 1994a,1994b). Patients with a VS caused by a TBI have better outcomes in terms of recovery of independence ( $24 \%$ versus $4 \%$ ) and consciousness $(52 \%$

\footnotetext{
${ }^{1}$ Unit for Severe Acquired Brain Injuries, ${ }^{2}$ Neurophysiology Unit, Rehabilitation Department, and ${ }^{5}$ Clinical Pathology and Microbiology Laboratory, Fondazione Istituto "San Raffaele-G. Giglio" Cefalù, Italy.

${ }^{3}$ Laboratorio di Tecnologie Oncologiche (LATO), HSR Giglio, Cefalù, Italy.

${ }^{4}$ Institute of Molecular Bioimaging and Physiology (IBFM), National Council of Research (CNR), Cefalù-Segrate, Italy.

${ }^{6}$ Research in Advanced Neuro-Rehabilitation, Sant'Anna Institute, Crotone, Italy.

${ }^{7}$ Cardinal Ferrari Center, Fontanellato, Italy.

${ }^{8}$ IRCCS S. Maugeri Foundation, Neurorehabilitation Unit, Scientific Institute of Pavia, Pavia, Italy.

${ }^{9}$ Post-Coma Unit and Headache Center, IRCCS Fondazione "Santa Lucia" Roma, Italy.

${ }^{10}$ Department of Sciences and Biomedical Technologies, UNIMI, Segrate, Milan, Italy.
} 
versus $13 \%$ ) than those with non-traumatic injuries (due to cerebral anoxia or stroke; Monti et al., 2010; Royal College of Physicians, 2003; The Multi-Society Task Force on PVS, 1994a,1994b). The reason for this improved outcome is not well understood. It may be related to different neuropathological findings. Indeed, the most common structural abnormality identified in patients in a VS after a TBI is a diffuse axonal injury, while in non-traumatic brain injuries diffuse ischemic damage in the neocortex is predominant (Adams et al., 2000). In addition, the better outcomes associated with TBIs may be related to the younger age of patients who typically suffer from TBIs (in comparison, for instance, to patients with vascular diseases or anoxia); notably, increased age worsens the recovery rate from a VS (The Multi-Society Task Force on PVS, 1994b). The third main factor related to consciousness improvement is the duration of VS itself, which correlates negatively with the chance of recovering consciousness (Braakman et al., 1998). These factors are valid in terms of group analyses. However, currently it remains extremely difficult to make a prognosis regarding consciousness and cognitive functional recovery in patients in a VS (Hirschberg and Giacino, 2011). This difficulty may at first be explained by the wide variety of brain injuries that can be caused during TBIs. Nevertheless, clinical practice suggests that additional factors are also probably involved in the outcomes for these patients. For example, neuroimaging studies have not uncovered specific features useful for predicting recovery from a VS, although some functional magnetic resonance imaging (fMRI) analyses may distinguish whether a patient is in a VS or not (Owen et al., 2006). The lack of correlation between neuroimaging studies and prognosis suggests that patients with similar brain injuries may have different outcomes.

Emergence from a VS implies that the brain undergoes changes in plasticity that restore connections and/or functions that existed prior to the traumatic event. Therefore, individual differences in genetic factors that regulate mechanisms underlying brain plasticity may hypothetically contribute to different outcomes. Polymorphisms in the gene encoding brain-derived neurotrophic factor (BDNF) are good candidates for playing this role. BDNF is a neurotrophin that performs a critical function in the modulation of synaptic efficacy (i.e., long-term potentiation [LTP]) involved in learning, memory, and adaptive behavior (Kleim et al., 2006; Poo, 2001; Tyler et al., 2002). Current knowledge concerning BDNF function shows that it is associated with intracellular signaling related to neuronal protection, synaptic function (glutamate-dependent spine and dendritic growth, synapse formation, stabilization, and potentiation), and morphological changes affecting neural development and function (Numakawa et al., 2010; Yoshii and Constantine-Paton, 2010). More specifically, in cellular and animal models, BDNF is involved in mechanisms underlying LTP induction and maintenance by activating latent synapses (Shen et al., 2006), and modulating cytoskeletal functions (Rex et al., 2007; Yano et al., 2006). The BDNF gene (gene ID 627 located on chromosome 11p13) has a common polymorphism (rs6265, dbSNP NCBI database), that leads to an amino acid substitution (valine to methionine) at codon 66 (Val66Met, referred to herein as the $\mathrm{BDNF}_{\text {Met }}$ polymorphism). This sequence variant is located in the $5^{\prime}$ pro-BDNF sequence, which encodes the precursor peptide (pro-BDNF), that is proteolytically cleaved to form the mature protein (Seidah et al., 1996).
Although this BDNF $_{\text {Met }}$ polymorphism does not affect transcription and translation processes or mature BDNF protein function, it has been shown to dramatically alter the intracellular trafficking and packaging of pro-BDNF, and thus the regulated secretion of the mature peptide (Chen et al., 2004). The $\mathrm{BDNF}_{\text {Met }}$ polymorphism is relatively common and is predicted to be present in 25-36\% of the Caucasian population (Pivac et al., 2009; Shimizu et al., 2004).

The $\mathrm{BDNF}_{\text {Met }}$ polymorphism is linked to some anatomical consequences in healthy subjects, and it has been associated with reduced hippocampal volume, decreased prefrontal cortex gray matter, and age-dependent modifications to the corpus callosum (Bueller et al., 2006; Nemoto et al., 2006; Pezawas et al., 2004; Szeszko et al., 2005). These findings have led to the hypothesis that BDNF-dependent plasticity may have some functional consequences in healthy subjects. Indeed, it has been demonstrated that the $\mathrm{BDNF}_{\text {Met }}$ polymorphism affects memory, cognitive function, and learning (Egan et al., 2003; Miyajima et al., 2008; Soliman et al., 2010).

BDNF may also influence the phenotypic expression of many diseases. For example, BDNF serum levels may be associated with cognitive impairment in schizophrenia (Zhang et al., 2012), or illness severity in major depression (Birkenhäger et al., 2012). Moreover, it has been shown that the $\mathrm{BDNF}_{\text {Met }}$ polymorphism may also affect recovery from hemorrhagic stroke such that it predicts a poor outcome among patients who survive an aneurysmal subarachnoid hemorrhage (Siironen et al., 2007). The BDNF Met $_{\text {polymor- }}$ phism has not been assessed previously in patients in a VS after a TBI, although it was recently demonstrated that other BDNF polymorphisms (rs7124442 and rs1519480) might be useful for predicting general intelligence 10-15 years after a penetrating TBI (Rostami et al., 2011).

Based on these findings, we evaluated whether the $\mathrm{BDNF}_{\text {Met }}$ polymorphism plays a role in the recovery of consciousness and cognitive function in patients in a VS after a TBI. To this end, we assessed the BDNF genotype in patients in VS 1 month after a TBI. We used the levels of cognitive function (LCF) scale (Hagen et al., 1972) to evaluate the recovery of consciousness and cognitive function. This scale is based on eight levels: levels 1 and 2 define patients in a VS, while the other levels denote a progressive improvement of cognitive function; level 8 describes patients with cognitive function close to their pre-morbid ability. We retrospectively correlated the different genotypes with consciousness levels evaluated with the LCF scale at 1, 3, and 6 months. The chances of regaining awareness in patients in a VS decrease as time passes, and recovery usually occurs within 6 months. However, in TBIs this recovery period lasts up to 12 months (Royal College of Physicians, 2003); therefore, we included also data at 12 months when available. The results of this study will help to clarify whether the $\mathrm{BDNF}_{\text {Met }}$ polymorphism is a useful prognostic factor in patients with VS after severe TBI.

\section{Materials and Methods}

\section{Patients}

This study was conducted with 53 patients who were in a VS after a TBI (45 males, 8 females; mean age $31.2 \pm 11.2$ years, range 15-55 years; all of Caucasian race), admitted to five Italian centers specializing in the rehabilitation of posttraumatic disorders of consciousness (Table 1). Four other 


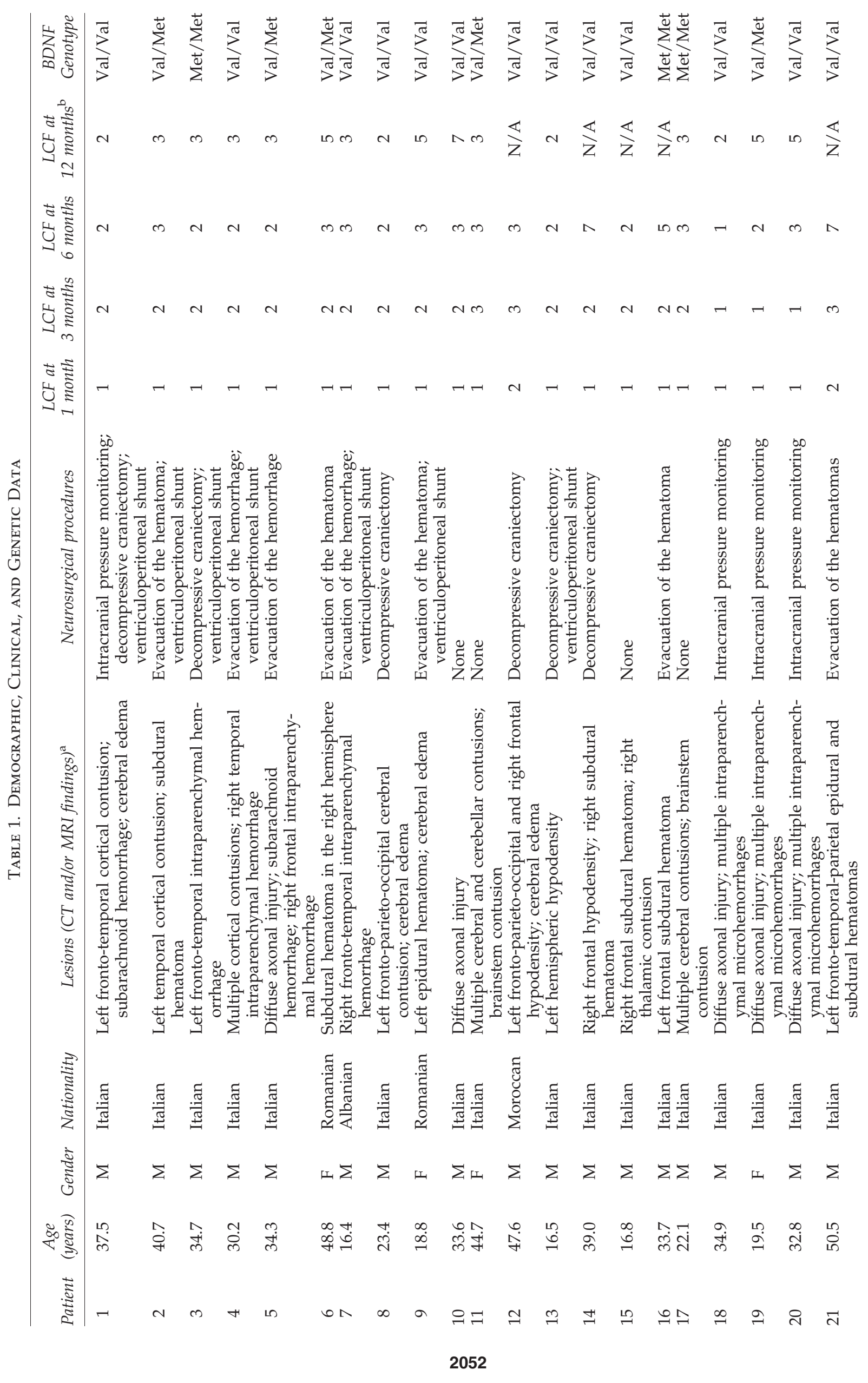




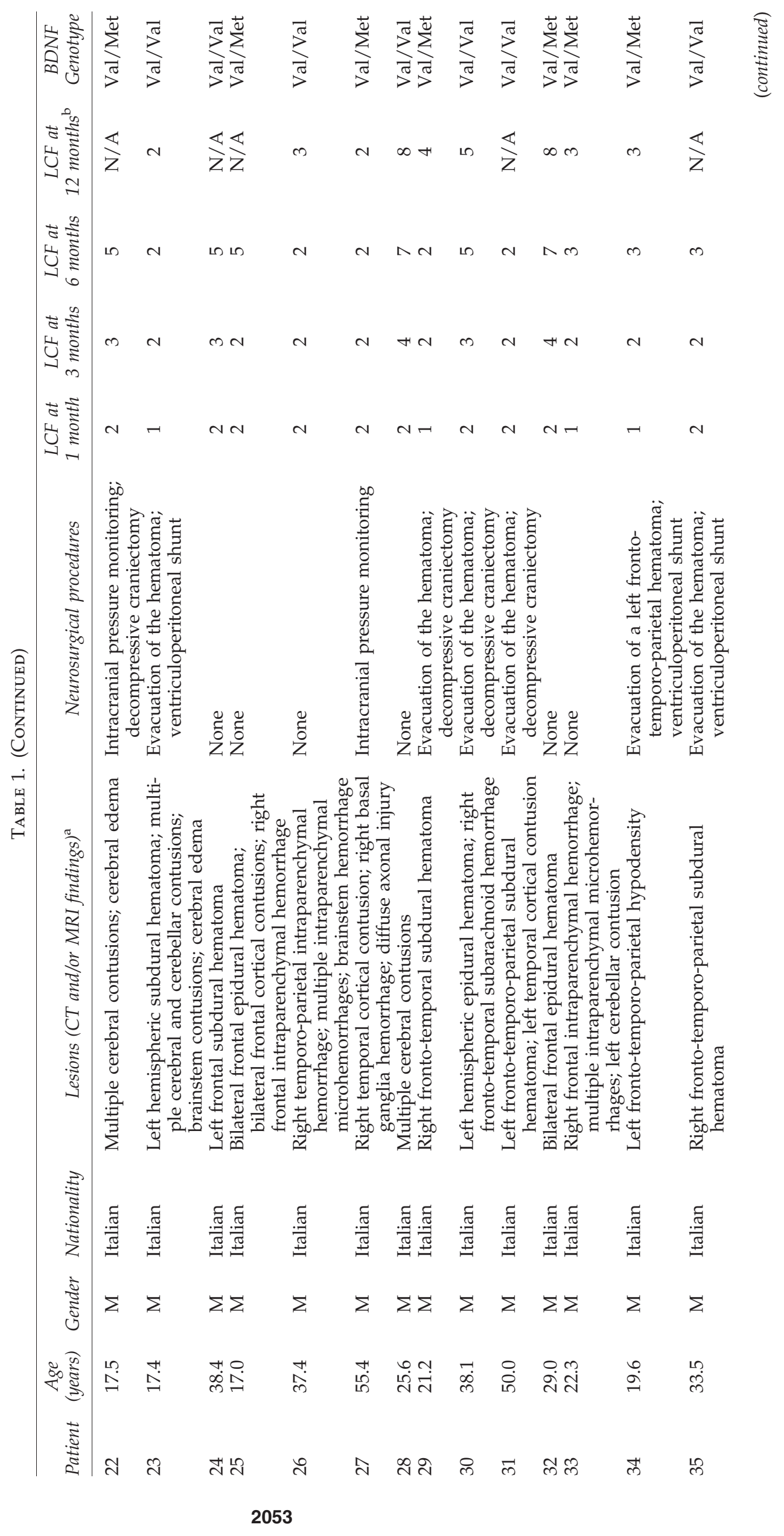




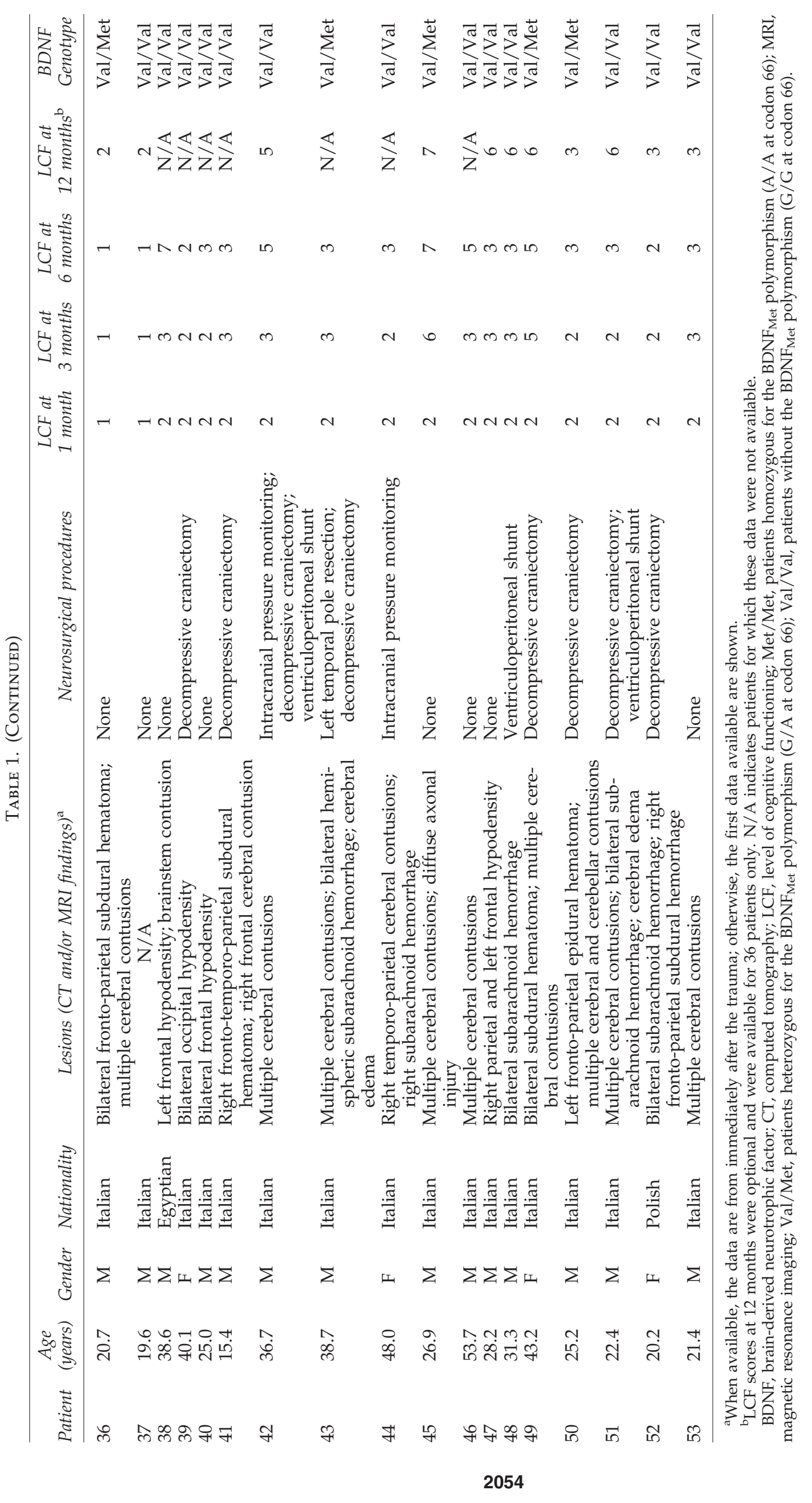


patients were excluded because of a lack of clinical data or informed consent. All of the patients underwent standard neurological/neurosurgical intensive care after the TBI. Thirtyfive patients underwent neurosurgical procedures, such as decompressive craniectomy (18 patients), hematoma evacuation (14 patients), ventriculoperitoneal shunt placement (13 patients), intracranial pressure monitoring (8 patients), and temporal pole resection (1 patient), alone or combined (Table 1). Inclusion criteria were the following: (1) age $\leq 55$ years at the time of the TBI (since people are conventionally considered elderly after 60 years of age, the age limit of 55 years was arbitrarily chosen in order to minimize the effects of the aging brain on recovery); (2) in a VS 1 month after TBI, according to current diagnostic criteria (Bates, 2005; Royal College of Physicians, 2003; The Multi-Society Task Force on PVS, 1994a); (3) LCF score of 1 or 2 at 1 month post-TBI; (4) available LCF scores at 1, 3 and 6 months post-TBI; and (5) no history of any neurological or psychiatric disease before the TBI.

The patients' relatives and/or legal guardians gave their written informed consent; the study and the consent procedure were performed according to the Helsinki Declaration, and were approved by the ethical committee of the coordinating center (Fondazione Istituto San Raffaele G. Giglio, Cefalù [PA], Italy; number of protocol: $\mathrm{BDNF} / 2010)$; the protocol was then ratified by the ethical committees of the other centers involved in the study.

\section{Clinical evaluation}

LCF scores at 1, 3, and 6 months were retrospectively obtained from clinical documentation; if the scores were not known with certainty, the patient was not included in the study. Clinical documentation of the LCF score at 12 months was available for 36 out of the 53 patients and these data were also analyzed. The diagnosis of VS and LCF assessments were made in all patients by a multidisciplinary team (neurologist, neuropsychologist, and speech therapist). Patients were included if the LCF scores at 1, 3, 6, and 12 months remained unchanged at the clinical evaluation performed on two consecutive days. Persistence of VS at 3, 6, and 12 months was defined according to clinical criteria (Bates, 2005; Royal College of Physicians, 2003; The Multi-Society Task Force on PVS, 1994a), and confirmed by an LCF score of 1 or 2 in all cases. The LCF scale defines eight levels of cognitive impairment (1: no response to stimuli; 2: generalized response; 3: localized response; 4: confused-agitated; 5: confused, inappropriate, nonagitated; 6: confused-appropriate; 7: automatic-appropriate; and 8: purposeful-appropriate; Hagen et al., 1972). All of the investigators collecting the clinical data were blinded to the results of the genetic analysis. Likewise, investigators who carried out the genetic analyses (L.M. and V.B.) were blinded to the patients' LCF scores. Finally, the statistical analyses were performed by a different researcher (S.B.).

\section{Genetic analysis}

Genotyping. Genetic analysis was performed on peripheral blood samples collected from five Italian centers after the patients were recruited. Blood samples were sent to the coordinating center (Rehabilitation Department, Fondazione Istituto "San Raffaele-G. Giglio," Cefalù, Italy), and then they were genotyped by the Laboratorio di Tecnologie Oncologiche (LATO)-HSR Giglio, Cefalù, Italy.
BDNF genotypes were assessed in all patients in order to identify carriers of the Val66Met polymorphism (rs6265) within the coding region of the BDNF gene, which is derived from a base substitution at codon $66(\mathrm{G} \rightarrow \mathrm{A})$. To genotype the $\mathrm{BDNF}_{\text {Met }}$ polymorphism, a restriction fragment length polymorphism (RFLP) assay and sequencing analysis were used. Total genomic DNA was extracted from peripheral blood using the QIAamp DNA blood mini-kit according to the manufacturer's specifications (Qiagen, Valencia, CA). Genomic DNA was PCR amplified using BDNF primers designed using Primer3 software (http//fokker.wi.mit.edu/primer3; BDNF forward: 5'-aaagaagcaaacatccgaggac-3' and BDNF reverse $5^{\prime}$-attcctccagcagaaagagaag- $\left.3^{\prime}\right)$. PCR reactions were performed using $50 \mathrm{ng}$ of genomic DNA in a total volume of $50 \mu \mathrm{L}$ containing $1 \times \mathrm{PCR}$ Gold Buffer, $1.5 \mathrm{mM}$ of $\mathrm{MgCl}_{2}$, $200 \mu \mathrm{M}$ dNTPs, $200 \mathrm{nM}$ of forward and reverse BDNF primer mix, and 1.25 U of AmpliTaq Gold DNA Polymerase (Applied Biosystems, Foster City, CA). The thermal cycle profile employed a 5 -min denaturing step at $94^{\circ} \mathrm{C}$, followed by 35 cycles at $94^{\circ} \mathrm{C}$ for $45 \mathrm{sec}, 58^{\circ} \mathrm{C}$ for $45 \mathrm{sec}, 72^{\circ} \mathrm{C}$ for $45 \mathrm{sec}$, and a final extension step of $5 \mathrm{~min}$ at $72^{\circ} \mathrm{C}$. The quality and concentration of the PCR products of $274 \mathrm{bp}$ were assessed using the Agilent 2100 Bioanalyzer according to the manufacturer's standard protocol (Agilent Technologies, Santa Clara, CA).

BDNFMet RFLP assay. An aliquot of the PCR product was digested with $10 \mathrm{U}$ of $H s p 92 \mathrm{II}$ restriction enzyme (RE) in a $50-\mu \mathrm{L}$ volume containing $1 \times \mathrm{RE}$ buffer and $5 \mu \mathrm{g}$ of bovine serum albumin (Promega, Fitchburg, WI) for $2 \mathrm{~h}$ at $37^{\circ} \mathrm{C}$. After heat inactivation by incubation at $65^{\circ} \mathrm{C}$ for $15 \mathrm{~min}$, the digested products were purified with the QIAquick PCR purification kit according to the manufacturer's specifications (Qiagen). RFLP analysis of Val66Met was carried out using the DNA 1000 kit in the Agilent 2100 Bioanalyzer. The Valhomozygous samples (G/G at codon 66) produced two visible products of $216 \mathrm{bp}$ and $58 \mathrm{bp}$, whereas Met-homozygous samples (A/A at codon 66) produced three visible products of $139 \mathrm{bp}, 77 \mathrm{bp}$, and $58 \mathrm{bp}$. Finally, heterozygous samples (G/A at codon 66) produced four fragments of $216 \mathrm{bp}, 139 \mathrm{bp}, 77 \mathrm{bp}$, and $58 \mathrm{bp}$.

BDNF sequencing. To confirm the RFLP genotyping data, the PCR products were sequenced. An aliquot of the PCR amplicon was purified using the QIAquick PCR purification kit according to the manufacturer's specifications (Qiagen). The concentration and quality of the DNA samples were determined using a NanoDrop ND-1000 Spectrophotometer. DNA sequencing was performed by MWG Biotech Laboratories (Ebersberg, Germany). In all cases, the sequencing results confirmed the previous data.

\section{Statistical analysis}

The patients were first divided into two groups (the Val group and the Met group) according to their genotype. Since the prevalence of the individuals homozygous for the $\mathrm{BDNF}_{\text {Met }}$ polymorphism is low among Caucasians (approximately 3.4\%; Pivac et al., 2009), the Met group included patients who were either heterozygous or homozygous for the $\mathrm{BDNF}_{\text {Met }}$ polymorphism. The preliminary sample size analysis was made considering an expected mean LCF value at 6 months of 3.3 for the Val group, an expected mean LCF 


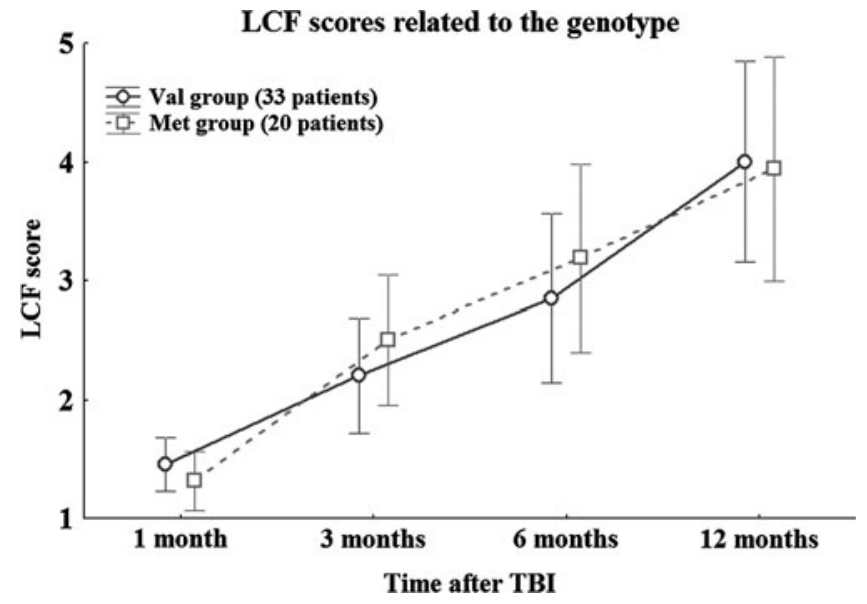

FIG. 1. LCF scores at 1, 3, 6, and 12 months post-TBI in patients without (Val group) and with (Met group) the $\mathrm{BDNF}_{\text {Met }}$ polymorphism. LCF scores at 12 months were optional, and available for 20 patients in the Val group and 16 patients in the Met group. Vertical bars denote $95 \%$ confidence intervals (LCF, level of cognitive functioning; TBI, traumatic brain injury; BDNF, brain-derived neurotrophic factor).

value of 2.5 for the Met group, a common standard deviation of 1.5, an alpha error level of 5\%, and a beta error level of $50 \%$. Based on these criteria, the required sample size was 38 patients (19 patients for each group). However, the effective sample size of the study was larger (53 patients).

Differences in demographic data were assessed by the Student's $t$-test. Fisher's exact test was used to evaluate whether the number of patients who emerged from a VS (i.e., with a LCF score $\geq 3$ ) at 3, 6, and 12 months was different in the Val and Met groups. Likewise, we compared the number of patients whose LCF scores improved at 3 months (with respect to 1 month), 6 months (with respect to 3 months), and
12 months (with respect to 6 months), in the Val and Met groups. Then the main statistical analysis was chosen to evaluate whether the BDNF genotypes influence LCF scores at $1,3,6$, and 12 months post-TBI. Thus, we used a two-way repeated-measures analysis of variance (ANOVA) with the factor group (Val group versus Met group) as the betweensubject factor, and the factor time (LCF score at 1 month versus LCF scores at 3, 6, and 12 months) as the within-subject factor. Mauchly's test was used to evaluate the sphericity of variances, and the Greenhouse-Geisser method was applied if necessary to correct for non-sphericity. Conditional on a significant $F$ value, post-hoc comparisons were performed using the Tukey honest significant difference test.

For all analyses, $p<0.05$ was considered statistically significant. Data are given as mean values \pm standard deviation.

\section{Results}

RFLP assays and sequencing analyses revealed the Val/Val genotype in 33 patients $(62.3 \%)$, whereas 20 patients $(37.7 \%)$ were carriers of the $\mathrm{BDNF}_{\text {Met }}$ polymorphism (including 17 heterozygotes and 3 homozygotes). The mean age was $31.5 \pm 11.2$ years in the Val group, and $30.8 \pm 11.4$ years in the Met group $(p=0.8)$. LCF scores at 12 months were optional, and data for analysis were available in 36 (67.9\%) of the 53 patients (20 in the Val group and 16 in the Met group, including 14 heterozygotes and 2 homozygotes).

Mean LCF scores in the Val and Met groups were 1.6 \pm 0.5 and $1.4 \pm 0.5$ at 1 month, $2.3 \pm 0.7$ and $2.5 \pm 1.2$ at 3 months, $3.3 \pm 1.7$ and $3.5 \pm 1.7$ at 6 months, and $4 \pm 1.9$ and $3.9 \pm 1.8$ at 12 months, respectively (Fig. 1). The percentages of patients who emerged from a VS in the Val and Met groups were $36.4 \%$ and $30 \%$ at 3 months $(p=0.8), 66.3 \%$ and $70 \%$ at 6 months $(p=0.8)$, and $70 \%$ and $87.5 \%$ at 12 months $(p=0.3)$, respectively (Table $2 \mathrm{~A})$. The percentages of patients who improved their LCF scores in the Val and Met groups were $67 \%$ and $75 \%$ at 3 months $(p=0.8), 48 \%$ and $60 \%$ at 6 months $(p=0.6)$, and $60 \%$ and $50 \%$ at 12 months $(p=0.7)$, respectively

Table 2. (A) Number of Patients Emerging (Yes) or Not (No) from a Vegetative State (VS) at 3, 6, and 12 Months after a Traumatic Brain Injury (TBI) in the Val and Met Groups. (B) Number of Patients Increasing (Yes) or Not (No) Their LCF Scores at 3, 6, and 12 Months after a TBI in the Val and Met Groups

\begin{tabular}{|c|c|c|c|c|c|c|c|c|c|}
\hline \multirow[b]{2}{*}{$A$} & \multicolumn{3}{|c|}{$\begin{array}{l}\text { Patients emerging from } \\
\text { a VS } 3 \text { months after a TBI }\end{array}$} & \multicolumn{3}{|c|}{$\begin{array}{l}\text { Patients emerging from } \\
\text { a VS } 6 \text { months after a TBI }\end{array}$} & \multicolumn{3}{|c|}{$\begin{array}{l}\quad \text { Patients emerging } \\
\text { from a VS } 12 \text { months after a TBI }\end{array}$} \\
\hline & Yes & No & Total & Yes & No & Total & Yes & No & Total \\
\hline Val group & 12 & 21 & 33 & 21 & 12 & 33 & 14 & 6 & 20 \\
\hline Met group & 6 & 14 & 20 & 14 & 6 & 20 & 14 & 2 & 16 \\
\hline Total & 18 & 35 & 53 & 35 & 18 & 53 & 28 & 8 & 36 \\
\hline \multirow[t]{2}{*}{ Fisher's exact test } & $p=0.8$ & & & $p=0.8$ & & & $p=0.3$ & & \\
\hline & \multicolumn{3}{|c|}{$\begin{array}{l}\text { Improvement of LCF score } \\
\text { at } 3 \text { months versus } 1 \text { month }\end{array}$} & \multicolumn{3}{|c|}{$\begin{array}{l}\text { Improvement of LCF score } \\
\text { at } 6 \text { months versus } 3 \text { months }\end{array}$} & \multicolumn{3}{|c|}{$\begin{array}{l}\text { Improvement of LCF score } \\
\text { at } 12 \text { months versus } 6 \text { months }\end{array}$} \\
\hline$B$ & Yes & No & Total & Yes & No & Total & Yes & No & Total \\
\hline Val group & 22 & 11 & 33 & 16 & 17 & 33 & 12 & 8 & 20 \\
\hline Met group & 15 & 5 & 20 & 12 & 8 & 20 & 8 & 8 & 16 \\
\hline Total & 37 & 16 & 53 & 28 & 25 & 53 & 20 & 16 & 36 \\
\hline Fisher's exact test & $p=0.8$ & & & $p=0.6$ & & & $p=0.7$ & & \\
\hline
\end{tabular}

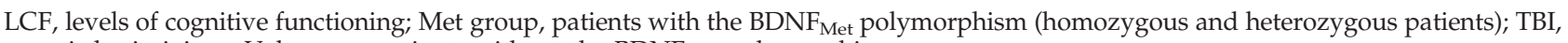
traumatic brain injury; Val group, patients without the $\mathrm{BDNF}_{\mathrm{Met}}$ polymorphism. 
(Table 2B). ANOVA showed a significant effect of the factor time $\left(\mathrm{F}_{1.9,64.6}=53.9 ; p<0.005\right)$, due to an overall improvement in LCF scores independent of the BDNF genotype $(p<0.05$ for LCF score at 3 months versus 1 month, LCF score at 6 months versus 3 months, and LCF score at 12 months versus 6 months). Indeed, the factor group $\left(\mathrm{F}_{1,34}=0.09 ; p=0.8\right)$, and the interaction group $\times$ time $\left(\mathrm{F}_{1.9,64.6}=0.7 ; p=0.5\right)$ did not show any significant differences between the Val and Met groups.

\section{Discussion}

In the current study, we provide evidence that the $\mathrm{BDNF}_{\mathrm{Met}}$ polymorphism is not involved in either recovery of consciousness or in the cognitive improvement of patients in VS after a severe TBI. Indeed, the percentages of patients who emerged from a VS at 3, 6, and 12 months after TBI did not differ between the Val and Met groups. Likewise, LCF scores at 1,3,6, and 12 months post-TBI were similar in both groups of patients, independent of the $\mathrm{BDNF}_{\mathrm{Met}}$ polymorphism.

To our knowledge, this is the first study evaluating the role of a genetic factor in the recovery of cognitive function in a large population of patients in a VS. Although the results rebut our initial hypothesis, some interesting considerations may be drawn from our data. Indeed, the $\mathrm{BDNF}_{\text {Met }}$ polymorphism does not seem to be involved in the cerebral mechanisms underlying cognitive improvement and emergence from a VS after a severe TBI. BDNF protein is widely distributed in the adult brain in almost all cortical areas, as well as in several subcortical and spinal cord regions (BakerHerman et al., 2004), and throughout life it influences the proliferation, differentiation, and functional activity of neuronal cells that underlie cognitive function (Kleim et al., 2006; Poo, 2001; Tyler et al., 2002). With regard to LTP, BDNFdependent mechanisms involved in cognitive tasks have been well studied, both in healthy subjects (Egan et al., 2003; Miyajima et al., 2008; Soliman et al., 2010), and in pathological conditions such as mood disorders (Chen et al., 2006). In particular, it has been reported that the $\mathrm{BDNF}_{\text {Met }}$ polymorphism may lead to a reduction in some cognitive functions in normal subjects (Hariri et al., 2003; Miyajima et al., 2008), or may predispose to major depression (Frodl et al., 2007), or anxiety disorders (Montag et al., 2010). However, unlike most other studies, we did not evaluate whether the $\mathrm{BDNF}_{\mathrm{Met}}$ polymorphism affects normal performance or predisposes to pathological conditions; rather, we investigated whether the $\mathrm{BDNF}_{\mathrm{Met}}$ polymorphism influences the recovery of cognitive function. In other words, the presence of the $\mathrm{BDNF}_{\mathrm{Met}}$ polymorphism may play distinct roles in the normal cognitive functions of healthy subjects (or predispose them to psychiatric disorders), and in the recovery of cognitive function after a severe TBI.

Although recovery from a VS may be considered a general process related to brain plasticity, a main factor that may explain the lack of correlation between BDNF genotype and outcomes of patients in a VS is the wide heterogeneity of brain injuries (see CT and MRI findings in Table 1). To reduce the variability in our population, we did not include patients who were in a VS due to a non-traumatic brain injury (e.g., patients in a VS after anoxic or vascular brain damage). Nevertheless, no two brain lesions associated with a TBI are identical, and there is a wide range of neuropathological findings in disabled head-injury survivors (Adams et al., 2011). Therefore, it may be useful to adopt even more stringent inclusion criteria and recruit patients with more homogeneous cerebral damage (for example, only those with a similar degree of diffuse axonal damage, or with brain injury due to anoxia) in future studies of correlations with genetic factors. In addition, we studied patients in a VS 1 month after TBI, even though unfavorable genetic factors may have already predisposed these patients to develop a VS after the comatose phase. Therefore, in future studies it may be interesting to genotype patients in an earlier phase (e.g., during a coma after a TBI), in order to evaluate whether some factors predispose them to develop a VS.

A potential limitation of our study is the scale we employed for our clinical evaluation. We chose the LCF scale because it was adopted by all participant centers and because it assesses only cognitive functions-and not, for instance, motor tasksthat were the target of the study. Moreover, the LCF scale has recently shown good correlation with some neurophysiological parameters in patients in a VS (Bagnato et al., 2010; Boccagni et al., 2011; Fingelkurts et al., 2012, in press). However, the LCF scale provides only an overall evaluation of cognitive functioning in these patients. Therefore it would be interesting to assess specific cognitive functions (e.g., memory, attention, and problem-solving) in patients recovering from a VS.

In the current study, carried out in Caucasian patients, we found a low occurrence rate of homozygosity for the BDNF polymorphism (3 patients, $5.5 \%$ of the total), which is in accord with its expected prevalence (3.4\%; Pivac et al., 2009). Therefore, as in other recent studies (Siironen et al., 2007; Soliman et al., 2010), we grouped patients with Val/Met and Met/Met genotypes. As a result, we cannot exclude the possibility that homozygous patients may have a worse outcome; this point should be further investigated in studies conducted in populations with a higher $\mathrm{BDNF}_{\text {Met }}$ polymorphism rate, such as Asian populations (Pivac et al., 2009).

Due to its widespread diffusion in the central nervous system, and on the basis of pathophysiological knowledge from animal models, BDNF has been proposed as a therapeutic tool in different neurological diseases such as Parkinson's disease, Alzheimer's disease, and spinal cord injuries (Blesch and Tuszynski, 2007; Nagahara et al., 2009; Tsukahara et al., 1995). However, the pathophysiology and physiological mechanisms underlying recovery in VS remain largely unknown, and animal models are not available to study this condition. The results of the current study provide evidence that the $\mathrm{BDNF}_{\text {Met }}$ polymorphism is not involved in the recovery of consciousness and cognitive improvement of patients in a VS after severe TBI. However, future studies should focus on the role of other BDNF polymorphisms in the cerebral reorganization associated with recovery in these patients.

\section{Acknowledgments}

For the collection of clinical data the authors wish to thank Beatrice Aiachini, M.D., Maugeri Foundation, Neurorehabilitation Unit, Scientific Institute of Pavia, Pavia, Italy; Paola Bossu, Ph.D., Clinical and Behavioural Neurology, IRCCS Fondazione "Santa Lucia," Roma, Italy; Serena Gennaro, M.D., Cardinal Ferrari Center, Fontanellato (PR), Italy; and Maria Girolama Raso, M.D., Research in Advanced NeuroRehabilitation, Sant'Anna Institute, Crotone, Italy. Special thanks also to all patients who participated to the study. 


\section{Author Disclosure Statement}

No competing financial interests exist.

\section{References}

Adams, J.H., Graham, D.I., and Jennett, B. (2000). The neuropathology of the vegetative state after acute brain insults. Brain 123, 1327-1338.

Adams, J.H., Jennett, B., Murray, L.S., Teasdale, G.M., Gennarelli, T.A., and Graham, D.I. (2011). Neuropathological findings in disabled survivors of a head injury. J. Neurotrauma 28, 701709.

Bagnato, S., Boccagni, C., Prestandrea, C., Sant'Angelo, A., Castiglione, A., and Galardi, G. (2010). Prognostic value of standard EEG in traumatic and non-traumatic disorders of consciousness following coma. Clin. Neurophysiol. 121, 274-280.

Baker-Herman, T.L., Fuller, D.D., Bavis, R.W., Zabka, A.G., Golder, F.J., Doperalski, N.J., Johnson, R.A., Watters, J.J., and Mitchell, G.S. (2004). BDNF is necessary and sufficient for spinal respiratory plasticity following intermittent hypoxia. Nat. Neurosci. 7, 48-55.

Bates, D. (2005). The vegetative state and the Royal College of Physicians guidance. Neuropsychol. Rehabil. 15, 175-183.

Birkenhäger, T.K., Geldermans, S., Van den Broek, W.W., van Beveren, N., and Fekkes, D. (2012). Serum brain-derived neurotrophic factor level in relation to illness severity and episode duration in patients with major depression. J. Psychiatr. Res. 46, 485-489.

Blesch, A., and Tuszynski, M.H. (2007). Transient growth factor delivery sustains regenerated axons after spinal cord injury. J. Neurosci. 27, 10535-10545.

Boccagni, C., Bagnato, S., Sant'Angelo, A., Prestandrea, C., and Galardi, G. (2011). Usefulness of standard EEG in predicting the outcome of patients with disorders of consciousness following anoxic coma. J. Clin. Neurophysiol. 28, 489-492.

Braakman, R., Jennett, W.B., and Minderhoud, J.M. (1998). Prognosis of the posttraumatic vegetative state. Acta Neurochirurgica (Wien.) 95, 49-52.

Bueller, J.A., Aftab, M., Sen, S., Gomez-Hassan, D., Burmeister, M., and Zubieta, J.K. (2006). BDNF Val66Met allele is associated with reduced hippocampal volume in healthy subjects. Biol. Psychiatry 59, 812-815.

Chen, Z.Y., Jing, D., Bath, K.G., Ieraci, A., Khan, T., Siao, C.J., Herrera, D.G., Toth, M., Yang, C., McEwen, B.S., Hempstead, B.L., and Lee, F.S. (2006). Genetic variant BDNF (Val66Met) polymorphism alters anxiety-related behavior. Science 314, 140-143.

Chen, Z.Y., Patel, P.D., Sant, G., Meng, C.X., Teng, K.K., Hempstead, B.L., and Lee, F.S. (2004). Variant brain-derived neurotrophic factor (BDNF) (Met66) alters the intracellular trafficking and activity-dependent secretion of wild-type BDNF in neurosecretory cells and cortical neurons. J. Neurosci. 24, 4401-4411.

Egan, M.F., Kojima, M., Callicott, J.H., Goldberg, T.E., Kolachana, B.S., Bertolino, A., Zaitsev, E., Gold, B., Goldman, D., Dean, M., Lu, B., and Weinberger, D.R. (2003). The BDNFval66met polymorphism affects activity-dependent secretion of BDNF and human memory and hippocampal function. Cell 11, 257-269.

Fingelkurts, A.A., Fingelkurts, A.A., Bagnato, S., Boccagni, C., and Galardi, G. (2012). EEG oscillatory states as neurophenomenology of consciousness as revealed from patients in vegetative and minimally conscious states. Conscious. Cogn. $21,149-169$.
Fingelkurts, A.A., Fingelkurts, A.A., Bagnato, S., Boccagni, C., and Galardi, G. (In press). The value of spontaneous EEG oscillations in distinguishing patients in vegetative and minimally conscious states. Supplements to Clinical Neurophysiology series, Volume 62.

Frodl, T., Schüle, C., Schmitt, G., Born, C., Baghai, T., Zill, P., Bottlender, R., Rupprecht, R., Bondy, B., Reiser, M., Möller, H.J., and Meisenzahl, E.M. (2007). Association of the brainderived neurotrophic factor Val66Met polymorphism with reduced hippocampal volumes in major depression. Arch. Gen. Psychiatry 64, 410-416.

Hagen, C., Malkmus, D., and Durham, P. (1972). Levels of Cognitive Functioning. Downey: Rancho Los Amigos Hospital.

Hariri, A.R., Goldberg, T.E., Mattay, V.S., Kolachana, B.S., Callicott, J.H., Egan, M.F., and Weinberger, D.R. (2003). Brainderived neurotrophic factor val66met polymorphism affects human memory-related hippocampal activity and predicts memory performance. J. Neurosci. 23, 6690-6694.

Hirschberg, R., and Giacino, J.T. (2011). The vegetative and minimally conscious states: diagnosis, prognosis and treatment. Neurol. Clin. 29, 773-786.

Kleim, J.A., Chan, S., Pringle, E., Schallert, K., Procaccio, V., Jimenez, R., and Cramer, S.C. (2006). BDNF val66met polymorphism is associated with modified experiencedependent plasticity in human motor cortex. Nat. Neurosci. 9, 735-737.

Miyajima, F., Ollier, W., Mayes, A., Jackson, A., Thacker, N., Rabbitt, P., Pendleton, N., Horan, M., and Payton, A. (2008). Brain-derived neurotrophic factor polymorphism Val66Met influences cognitive abilities in the elderly. Genes Brain Behav. 7, 411-417.

Montag, C., Basten, U., Stelzel, C., Fiebach, C.J., and Reuter, M. (2010). The BDNF Val66Met polymorphism and anxiety: support for animal knock-in studies from a genetic association study in humans. Psychiatry Res. 179, 86-90.

Monti, M.M., Laureys, S., and Owen, A.M. (2010). The vegetative state. B.M.J. 341, c3765.

Nagahara, A.H., Merrill, D.A., Coppola, G., Tsukada, S., Schroeder, B.E., Shaked, G.M., Wang, L., Blesch, A., Kim, A., Conner, J.M., Rockenstein, E., Chao, M.V., Koo, E.H., Geschwind, D., Masliah E., Chiba, A.A., and Tuszynski, M.H. (2009). Neuroprotective effects of brain-derived neurotrophic factor in rodent and primate models of Alzheimer's disease. Nat. Med. 15, 331-337.

Nemoto, K., Ohnishi, T., Mori, T., Moriguchi, Y., Hashimoto, R., Asada, T., and Kunugi, H. (2006). The Val66Met polymorphism of the brain-derived neurotrophic factor gene affects age-related brain morphology. Neurosci. Lett. 397, 25-29.

Numakawa, T., Suzuki, S., Kumamaru, E., Adachi, N., Richards, M., and Kunugi, H. (2010). BDNF function and intracellular signaling in neurons. Histol. Histopathol. 25, 237-258.

Owen, A.M., Coleman, M.R., Boly, M., Davis, M.H., Laureys, S., and Pickard, J.D. (2006). Detecting awareness in the vegetative state. Science 313, 1402.

Pezawas, L., Verchinski, B.A., Mattay, V.S., Callicott, J.H., Kolachana, B.S., Straub, R.E., Egan, M.F., Meyer-Lindenberg, A., and Weinberger, D.R. (2004). The brain-derived neurotrophic factor val66met polymorphism and variation in human cortical morphology. J. Neurosci. 24, 10099-10102.

Pivac, N., Kim, B., Nedić, G., Joo, Y.H., Kozarić-Kovacić, D., Hong, J.P., and Muck-Seler, D. (2009). Ethnic differences in brain-derived neurotrophic factor Val66Met polymorphism in Croatian and Korean healthy participants. Croat. Med. J. 50, 43-48. 
Poo, M.M. (2001). Neurotrophins as synaptic modulators. Nat. Rev. Neurosci. 2, 24-32.

Rex, C.S., Lin, C.Y., Kramar, E.A., Chen, L.Y., Gall, C.M., and Lynch, G. (2007). Brain-derived neurotrophic factor promotes long-term potentiation-related cytoskeletal changes in adult hippocampus. J. Neurosci. 27, 3017-3029.

Rostami, E., Krueger, F., Zoubak, S., Dal Monte, O., Raymont, V., Pardini, M., Hodgkinson, C.A., Goldman, D., Risling, M., and Grafman, J. (2011). BDNF polymorphism predicts general intelligence after penetrating traumatic brain injury. PLoS One 6, e27389.

Royal College of Physicians. (2003). The permanent vegetative state: guidance on diagnosis and management. Report of a working party. R.C.P. http://bookshop.rcplondon.ac.uk/ contents /47a262a7-350a-490a-b88d-6f58bbf076a3.pdf

Seidah, N.G., Benjannet, S., Pareek, S., Chretien, M., and Murphy, R.A. (1996). Cellular processing of the neurotrophin precursors of NT3 and BDNF by the mammalian proprotein convertases. FEBS Lett. 379, 247-250.

Shen, W., Wu, B., Zhang, Z., Dou, Y., Rao, Z.R., Chen, Y.R., and Duan, S. (2006). Activity-induced rapid synaptic maturation mediated by presynaptic cdc42 signaling. Neuron 50, 401-414.

Shimizu, E., Hashimoto, K., and Iyo, M. (2004). Ethnic difference of the BDNF 196G/A (val66met) polymorphism frequencies: the possibility to explain ethnic mental traits. Am. J. Med. Genet. B Neuropsychiatr. Genet. 126B, 122-123.

Siironen, J., Juvela, S., Kanarek, K., Vilkki, J., Hernesniemi, J., and Lappalainen, J. (2007). The Met allele of the BDNF Val66Met polymorphism predicts poor outcome among survivors of aneurysmal subarachnoid hemorrhage. Stroke 38, 2858-2860.

Soliman, F., Glatt, C.E., Bath, K.G., Levita, L., Jones, R.M., Pattwell, S.S., Jing, D., Tottenham, N., Amso, D., Somerville, L.H., Voss, H.U., Glover, G., Ballon, D.J., Liston, C., Teslovich, T., Van Kempen, T., Lee, F.S., and Casey, B.J. (2010). A genetic variant BDNF polymorphism alters extinction learning in both mouse and human. Science 327, 863-866.

Szeszko, P.R., Lipsky, R., Mentscheln, C., Robinson D., GunduzBruce, H., Sevy, S., Ashtari, M., Napolitano, B., Bilder, R.M., Kane, J.M., Goldman, D., and Malhotra, A.K. (2005). Brain- derived neurotrophic factor val66met polymorphism and volume of the hippocampal formation. Mol. Psychiatry 10, 631-636.

Tang, S., Machaalani, R., and Waters, K.A. (2010). Immunolocalization of pro- and mature-brain derived neurotrophic factor (BDNF) and receptor TrkB in the human brainstem and hippocampus. Brain Res. 1354, 1-14.

The Multi-Society Task Force on PVS. (1994a). Medical aspects of the persistent vegetative state (1). N. Engl. J. Med. 330, 14991508 .

The Multi-Society Task Force on PVS. (1994b). Medical aspects of the persistent vegetative state (2). N. Engl. J. Med. 330, 1572-1579.

Tsukahara, T., Takeda, M., Shimohama, S., Ohara, O., and Hashimoto, N. (1995). Effects of brain-derived neurotrophic factor on 1-methyl-4-phenyl-1,2,3,6-tetrahydropyridine-induced parkinsonism in monkeys. Neurosurgery 37, 733-739.

Tyler, W.J., Alonso, M., Bramham, C.R., and Pozzo-Miller, L.D. (2002). From acquisition to consolidation: on the role of brainderived neurotrophic factor signaling in hippocampal-dependent learning. Learn. Mem. 9, 224-237.

Yano, H., Ninan, I., Zhang, H., Milner, T.A., Arancio, O., and Chao, M.V. (2006). BDNF-mediated neurotransmission relies upon a myosin VI motor complex. Nat. Neurosci. 9, 1009-1018. Yoshii, A., and Constantine-Paton, M. (2010). Postsynaptic BDNF-TrkB signaling in synapse maturation, plasticity, and disease. Dev. Neurobiol. 70, 304-322.

Zhang, X.Y., Liang, J., Chen, D.C., Xiu, M.H., De Yang, F., Kosten, T.A., and Kosten T.R. (2012). Low BDNF is associated with cognitive impairment in chronic patients with schizophrenia. Psychopharmacology (Berl.) 222, 277-284.

Address correspondence to: Sergio Bagnato, M.D., Ph.D.

Unit for Severe Acquired Brain Injuries Rehabilitation Department Fondazione Istituto "San Raffaele-G. Giglio" 90015 Cefalù (PA), Italy

E-mail: sergiobagnato@gmail.com 